\title{
TITLE:
}

\section{Analytic continuations of de Sitter thick domain wall solutions}

AUTHOR(S):

Sasakura, N

CITATION:

Sasakura, N. Analytic continuations of de Sitter thick domain wall solutions. PHYSICAL REVIEW D 2002, 66(6): 065006.

ISSUE DATE:

2002-09-15

URL:

http://hdl.handle.net/2433/50461

RIGHT:

Copyright 2002 American Physical Society 
PHYSICAL REVIEW D 66, 065006 (2002)

\title{
Analytic continuations of de Sitter thick domain wall solutions
}

\author{
Naoki Sasakura* \\ Yukawa Institute for Theoretical Physics, Kyoto University, Kyoto 606-8502, Japan
}

(Received 12 March 2002; published 20 September 2002)

\begin{abstract}
I perform some analytic continuations of the de Sitter thick domain wall solutions obtained in my previous paper in the system of gravity and a scalar field with an axionlike potential. The obtained new solutions represent anti-de Sitter thick domain walls and cosmology. The anti-de Sitter domain wall solutions are periodic, and correspondingly the cosmological solutions represent cyclic universes. I parametrize the axionlike scalar field potential and determine the parameter regions of each type of solutions.
\end{abstract}

DOI: 10.1103/PhysRevD.66.065006

PACS number(s): 11.27.+d, 04.20.Jb, 04.50.+h

\section{INTRODUCTION}

The solitonic objects in string theory play major roles in the understanding of its nonperturbative properties such as dualities. In string theory the solitons couple necessarily with gravity. In view of the universality of the gravitational interaction, the understanding of the gravitational aspects of solitons may provide a deeper understanding of the variety of the dynamics contained in string theory.

The simplest system of solitons and gravity would be domain walls of scalar field theory interacting with gravity. In field theory, domain walls appear when there are more than one vacua in a scalar field potential, and it is a straightforward matter to obtain static domain wall configurations. On the other hand, with gravitational interactions, their nonlinearity and instability make it a nontrivial issue to study the dynamics of domain walls. As for a supersymmetric domain wall configuration, it can be analyzed by Bogomol'nyiPrasad-Sommerfield (BPS) first order differential equations [2]. These solutions are static and it was shown that a generic static and flat domain wall configuration can be analyzed in the same manner [3-6]. The procedure to obtain such a solution is mathematically well defined, but an obtained solution is not necessarily physically meaningful. In fact, the scalar field potential must be fine-tuned to avoid naked curvature singularities $[1,6-8]$. This situation is quite unsatisfactory, since a scalar field potential will change its form from a supersymmetric one by possible low energy dynamics such as supersymmetry breaking and instanton corrections, even if I assume some high energy supersymmetries. The nonsupersymmetric string theories also suffer from a similar pathological behavior that the background solutions have naked singularities where the dilaton field diverges $[9,10]$.

One of the possible resolutions to these singularities is to introduce time dependences of domain walls. The use of a de Sitter expansion to turn a curvature singularity of a static soliton into a horizon has appeared in the context of global $U(1)$ vortex solutions [11] and in a codimension two nonsupersymmetric soliton solution in type IIB string theory [12]. This is also used in constructing background solutions of nonsupersymmetric string theories [13]. As for timedependent domain walls, perturbative analyses have been

\footnotetext{
*Electronic address: sasakura@yukawa.kyoto-u.ac.jp
}

performed for the system of gravity and a scalar field in $[14,15]$. The constructions of analytic de Sitter thick domain wall solutions with horizons were done in $[1,16]$.

In my previous paper [1], the scalar field potential takes an axionlike form and its two parameters are restricted to a certain region for the analytic solutions to exist. The motivation of this paper is to find analytical solutions for the outside of this region of these parameters. It is a well known trick that, starting from a domain wall solution, analytic continuations generate domain wall solutions with flipped curvatures and cosmological solutions [17]. I will show that the new solutions cover the missing parameter regions. They are anti-de Sitter domain walls, finite lifetime universes with a big-bang and a big-crunch, and cyclic universes.

\section{II. de SITTER DOMAIN WALL SOLUTIONS}

In my previous paper [1], I obtained a class of analytic solutions of thick domain walls with de Sitter expansions in the system of five-dimensional gravity and a scalar field with an axion-like potential. Let me start my discussions by extending my previous results to a general space-time dimension $n$.

The action of my system is given by

$$
S=\int d t d x^{n-2} d y \sqrt{-g}\left(R-\frac{1}{2} g^{\mu \nu} \partial_{\mu} \phi \partial_{\nu} \phi-V(\phi)\right) .
$$

The metric ansatz I use is the warped geometry

$$
d s^{2}=a(y)^{2}\left(-d t^{2}+e^{2 H t} \sum_{i=1}^{n-2}\left(d x^{i}\right)^{2}\right)+d y^{2}
$$

where $H$ denotes the Hubble constant of the $(n-1)$-dimensional de Sitter space-time. Under the assumption that the scalar field depends only on the coordinate $y$, the Einstein equations are

$$
\left(\phi^{\prime}\right)^{2}=\frac{2(n-2)\left[\left(a^{\prime}\right)^{2}-a a^{\prime \prime}-H^{2}\right]}{a^{2}},
$$



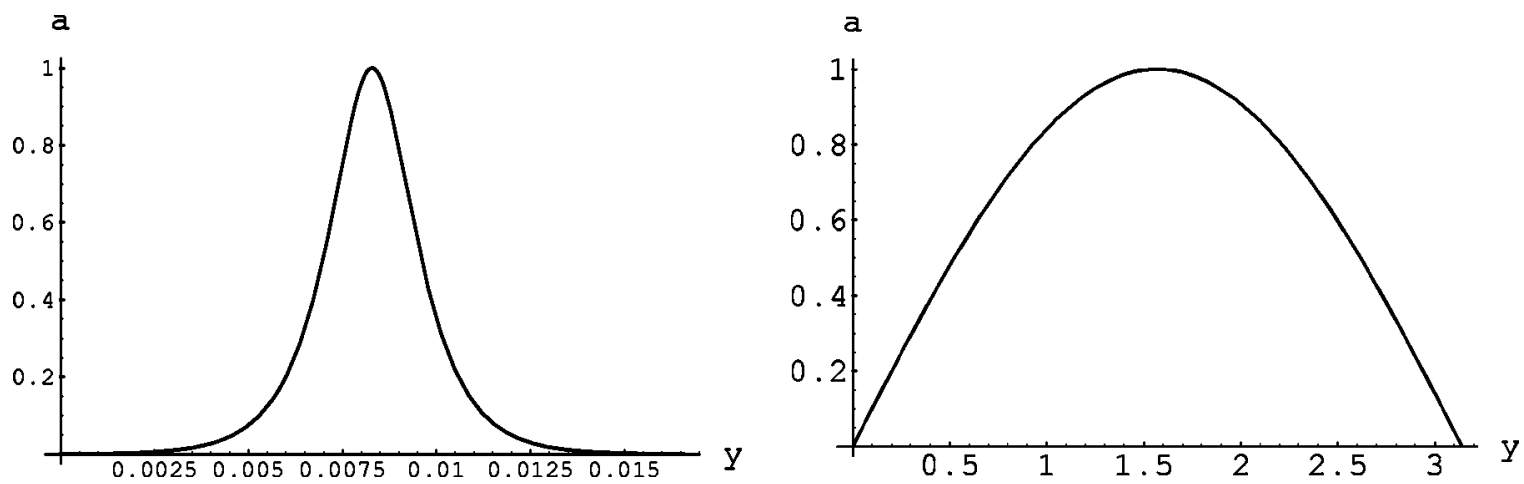

FIG. 1. The shapes of the de Sitter domain walls with $H=1$ and $\beta=0.001$ and $\beta=1000$.

$$
V(\phi)=\frac{(n-2)\left[-a a^{\prime \prime}-(n-2)\left(a^{\prime}\right)^{2}+(n-2) H^{2}\right]}{a^{2}},
$$

where the prime denotes the derivation with respect to $y$. The equation of motion of the scalar field is automatically satisfied by the solutions of Eq. (3) because of a Bianchi identity. By repeating the same procedure as done in my previous paper [1], I find that the first equation of Eq. (3) is satisfied by the solution

$$
\begin{aligned}
& a(y)=\operatorname{sn}\left(H y, i \beta^{-1}\right), \\
& \phi(y)=\sqrt{2(n-2)} \arctan \left(\frac{\operatorname{cn}\left(H y, i \beta^{-1}\right)}{\beta \operatorname{dn}\left(H y, i \beta^{-1}\right)}\right),
\end{aligned}
$$

where the elliptic functions are defined by

$$
\operatorname{sn}^{-1}(z, k)=\int_{0}^{z} \frac{d x}{\sqrt{\left(1-x^{2}\right)\left(1-k^{2} x^{2}\right)}}
$$

$\operatorname{cn}(u, k)=\left[1-\operatorname{sn}^{2}(u, k)\right]^{1 / 2} \quad$ and $\quad \operatorname{dn}(u, k)=[1$ $\left.-k^{2} \operatorname{sn}^{2}(u, k)\right]^{1 / 2}$. Here the parameter $\beta$ is a free real parameter. In the expression (4), the normalization of the scale factor $a(y)$ merely defines the unit of length scale and I have normalized it by imposing $a=1$ at the domain wall peak defined by $a^{\prime}=0$, and the constant shift ambiguity of $\phi(y)$ is fixed by imposing $\phi=0$ at the domain wall peak. A peculiar property of the solution (3) is that the scale factor behaves near its vanishing point $y=0$ as

$$
a(y)=H y+O\left(y^{3}\right) .
$$

As discussed in my previous paper [1], this behavior is required for the vanishing point to be regular. The factor $H$ of the linear term can be also understood from the physical consistency of the geometry that the temperature associated to the Rindler space-time near $y=0$ should agree with that of the de Sitter domain wall space-time $H / 2 \pi$ (see Fig. 1). I will show in Sec. III that the vanishing point is actually a horizon and that a regular extended space-time can be obtained by taking an appropriate coordinate system.

The scalar field potential is determined by the second equation of Eq. (3), and we obtain

$$
\begin{aligned}
V(\phi)= & \frac{H^{2}(n-2)^{2}\left(1-\beta^{-2}\right)}{2} \\
& +\frac{H^{2} n(n-2)\left(1+\beta^{-2}\right)}{2} \cos \left(\sqrt{\frac{2}{n-2}} \phi\right),
\end{aligned}
$$

which is similar to that of an axion with an instanton correction. In this paper I consider the two-dimensional parameter space $\left(v_{0}, v_{1}\right)$ of the scalar field potential,

$$
V(\phi)=v_{0}+v_{1} \cos \left(\sqrt{\frac{2}{n-2}} \phi\right) .
$$

I may assume $v_{1}$ to be a positive parameter by shifting $\phi$, appropriately. In this parametrization the result (7) shows that the parameter region for the existence of a regular de Sitter domain wall solution is given by

$$
-(n-2) v_{1}<n v_{0}<(n-2) v_{1} \text {. }
$$

\section{EXTENSION OF THE de SITTER DOMAIN WALL SPACE-TIME}

The vanishing points $a=0$ are actually horizons. To show this I will extend the solutions (4). The extended space-time is essentially equivalent to the $n=1 / 2$ case of [16], and I will follow their discussions. I first change to a conformal coordinate $d z=d y / a$. Integrating the solution (4), I obtain

$$
z-z_{0}=\frac{1}{H} \ln \left(\frac{\operatorname{sn}\left(H y, i \beta^{-1}\right)}{\operatorname{dn}\left(H y, i \beta^{-1}\right)+\operatorname{cn}\left(H y, i \beta^{-1}\right)}\right),
$$

where $z_{0}$ is an integration constant. Using Eq. (10) and taking a proper value of $z_{0}$, the metric for a de Sitter domain wall solution becomes

$$
\begin{aligned}
d s^{2}= & \frac{2}{\left(1+\beta^{-2}\right) \cosh (2 H z)+1-\beta^{-2}}\left(-d \tau^{2}+d z^{2}\right. \\
& \left.+H^{-2} \cosh ^{2}(H \tau) d \Omega^{n-2}\right)
\end{aligned}
$$

where I have changed to a global coordinate of a de Sitter space-time instead of a flat one appearing in Eq. (2), and 


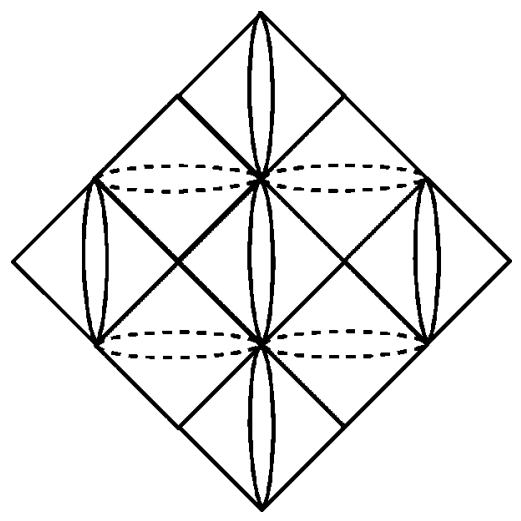

FIG. 2. The Penrose diagram of the de Sitter domain wall spacetime. The two edges of each oval should be identified. The solid ones depict the peaks of the domain walls, while the dashed ones the bottoms.

$d \Omega^{n-2}$ denotes the metric on an $(n-2)$-dimensional unit sphere. By a further transformation of $u=\exp [H(\tau-z)]$ and $v=\exp [-H(\tau+z)]$, I obtain

$$
\begin{aligned}
d s^{2}= & \frac{4 H^{-2}}{\left(1+\beta^{-2}\right)\left(1+u^{2} v^{2}\right)+2\left(1-\beta^{-2}\right) u v}(d u d v \\
& \left.+\left(\frac{u+v}{2}\right)^{2} d \Omega^{n-2}\right) .
\end{aligned}
$$

Note that the allover factor of the metric (12) is nonsingular for all the real values of $u$ and $v$. After the change of variables $u=T+R, v=-T+R$, the metric in the parentheses becomes $-d T^{2}+d R^{2}+R^{2} d \Omega^{n-2}$ and is free of a conical singularity. As pointed out in [16], the domain wall peak at $-T^{2}+R^{2}=u v=1$ is a bubble with a constant acceleration in the coordinate $(T, R, \Omega)$ (see Fig. 2 ).

As for the scalar field, I obtain

$$
\phi=\sqrt{2(n-2)} \arctan \left(\frac{u v-1}{\beta(u v+1)}\right),
$$

which is also well defined for any real values of $u$ and $v$.

\section{ANTI-de SITTER DOMAIN WALL SOLUTIONS}

In the following I will study the other parameter regions of the potential (8) rather than (9). Looking at the expression (7), one notices that all the other parameter regions of $v_{0}$ and $v_{1}$ can be covered by the analytic continuations of the parameters $\beta$ and $H$ to pure imaginary values. However, under an analytic continuation of only one of $\beta$ or $H$ to a pure imaginary value, one of $\phi(y)$ or $a(y)$ becomes imaginary and physically meaningless. This cannot be resolved even by using the constant shift ambiguity of $y$ of the solution. To obtain a physically meaningful expression from Eq. (4), both $\beta$ and $H$ must be analytically continued to imaginary values, and, after some trials, it turns out that the analytic continua- tion should contain a simultaneous shift of $y$ as $^{1}$

$$
\begin{gathered}
1 / \beta \rightarrow-i \delta, \\
H \rightarrow i h, \\
H y \rightarrow i h y+K(\delta),
\end{gathered}
$$

where $\delta$ and $h$ are real constants and $K(\delta)$ is the elliptic integral of the first kind,

$$
K(\delta)=\int_{0}^{1} \frac{d x}{\sqrt{\left(1-x^{2}\right)\left(1-\delta^{2} x^{2}\right)}}
$$

Substituting the analytic continuation (14) into the de Sitter solution (4), I obtain new solutions

$$
\begin{aligned}
& a(y)=\frac{1}{\operatorname{dn}\left(h y, \sqrt{1-\delta^{2}}\right)}, \\
& \phi(y)=\sqrt{2(n-2)} \arctan \left(\frac{\delta \operatorname{sn}\left(h y, \sqrt{1-\delta^{2}}\right)}{\operatorname{cn}\left(h y, \sqrt{1-\delta^{2}}\right)}\right),
\end{aligned}
$$

and

$$
\begin{aligned}
V(\phi)= & -\frac{h^{2}(n-2)^{2}\left(1+\delta^{2}\right)}{2} \\
& -\frac{h^{2} n(n-2)\left(1-\delta^{2}\right)}{2} \cos \left(\sqrt{\frac{2}{n-2}} \phi\right) .
\end{aligned}
$$

By the parametrization (8), the scalar potential (17) is in the region

$$
n v_{0}<-(n-2) v_{1} .
$$

To make the warped metric (2) meaningful under the analytic continuation (14), I perform a simultaneous continuation $t \rightarrow i r$ and $x^{1} \rightarrow i x^{0}$. Then Eq. (2) becomes an anti-de Sitter domain wall metric

$$
\begin{aligned}
d s^{2}= & a(y)^{2}\left(d r^{2}+e^{-2 h r}\left(-\left(d x^{0}\right)^{2}+\sum_{i=2}^{n-2}\left(d x^{i}\right)^{2}\right)\right) \\
& +d y^{2}
\end{aligned}
$$

Thus in the region (18), there exist regular AdS domain wall solutions, which are given by Eqs. (16) and (17) (see Fig. 3).

The stability of the AdS solutions can be checked as follows. I restrict my attention to the five-dimensional case ( $n$ $=5$ ). Presumably the extension to a general dimension will be straightforward. As discussed in $[6,7,18,19]$, the problem of obtaining the mass spectra of the linear perturbations around a solution boils down to solving Schrödinger equations. As for the tensor perturbation,

\footnotetext{
${ }^{1}$ The other available choices of the shift of $y$ do not give any other independent solutions.
} 


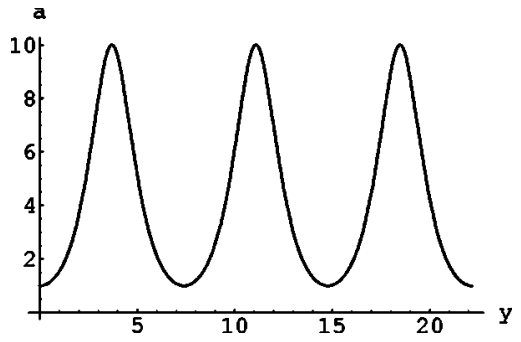

FIG. 3. The shape of the anti-de Sitter domain wall with $h$ $=1$ and $\delta=0.1$.

$$
\begin{aligned}
d s^{2} & =a^{2}\left[\left(\gamma_{\mu \nu}+h_{\mu \nu}\right) d x^{\mu} d x^{\nu}+d z^{2}\right], \\
h_{\mu}^{\mu} & =h_{\mu \nu}^{\mid \nu}=0,
\end{aligned}
$$

the Schrödinger equation has a supersymmetric form

$$
Q^{\dagger} Q \varphi=m^{2} \varphi
$$

where

$$
Q=\frac{d}{d z}-\frac{3}{2 a} \frac{d a}{d z}
$$

Thus the eigenvalue $m^{2}$ is non-negative, and hence the stability under the tensor perturbations is satisfied. As for the scalar perturbation,

$$
d s^{2}=a^{2}\left[\left(1+\psi_{1}\right) \gamma_{\mu \nu} d x^{\mu} d x^{\nu}+\left(1+\psi_{2}\right) d z^{2}\right]
$$

the Schrödinger equation becomes $[18,19,1]$

$$
\left(\widetilde{Q}^{\dagger} \widetilde{Q}+\frac{a^{2}\left(\phi^{\prime}\right)^{2}}{3}+4 h^{2}\right) \varphi=m^{2} \varphi,
$$

where

$$
\widetilde{Q}=\frac{d}{d z}+\frac{d \ln \left(a^{3 / 2} \phi^{\prime}\right)}{d z}
$$

This Schrödinger equation is obtained by the substitution $H$ $\rightarrow i h$ in the corresponding expression in [1]. The $m^{2}$ is obviously positive, and the AdS solutions are stable under the scalar perturbations. However, there remains one thing to check before this conclusion. If there existed points with $\phi^{\prime}=0$, the operator $\widetilde{Q}$ would become singular at these points and the above naive discussion of the positivity would be in danger. Moreover, in the derivation of the Schrödinger equation for the scalar perturbations in [19], the linear perturbation is redefined by a multiplication of a factor which is singular if $\phi^{\prime}=0$. But, in the solution (16), $\phi^{\prime}$ is always nonzero, and the above discussion is safe.

\section{THE REGION WITHOUT REGULAR SOLUTIONS}

I cannot find a physically meaningful analytic continuation to the parameter region

$$
(n-2) v_{1}<n v_{0} \text {. }
$$

Thus it is suspected that there are no regular domain wall solutions of the form of the warped metric (2) in this parameter region. To see whether this is the case, I will use a numerical computation.

Since, in this parameter region, the constant part $v_{0}$ of the scalar potential is larger than that of the parameter region of de Sitter analytic solutions (9), I may assume the solution to be a de Sitter domain wall rather than an anti-de Sitter one. As generally discussed in my previous paper [1], a regular de Sitter domain wall solution is sandwiched between two horizons. The initial values of the differential equations (3) can be provided by the three values $a, a^{\prime}$, and $\phi$ at a certain initial location of $y$. For the solution to be regular at a horizon, the scale factor must behave in the form (6), and therefore the freedom to choose the initial values is reduced to the only free parameter $\phi=\phi_{c}$ at the horizon. Thus, to search for a regular solution, I take one of the horizons as the initial location and integrate numerically the differential equations (3) for each value of $\phi_{c}$ at the horizon. Then the question of the existence of a regular solution is translated to whether there exists an initial value $\phi_{c}$ for which the numerical solution of the differential equations is regular between the initial horizon and the other horizon of a domain wall.

This search procedure gives a numerical regular solution for each choice of $\left(v_{0}, v_{1}, H\right)$. Studying in this threedimensional space would be too much, and in fact, I can reduce the dimension to one by rescaling the differential equations (3). By the rescaling of $y$ and $a$, I can normalize the scalar potential and the Hubble constant so that $v_{1}=1$ and $H=1$. Thus it is enough to check the question for each choice of $v_{0}$.

It would be a reasonable assumption that a domain wall contains the peak of the potential energy $\phi=0$. Then, since the potential (8) is $Z_{2}$ invariant, ${ }^{2}$ it is enough for me to solve the equations in the region $\phi>0$. I take an initial value $\phi$ $=\phi_{c}>0$ and solve the differential equations (3) taking the branch $\phi^{\prime}<0$ until the solution reaches the value $\phi=0$. By sweeping the initial value $\phi_{c}$, I obtain the range of $a^{\prime} / a$ at $\phi=0$. If the obtained range of $a^{\prime} /\left.a\right|_{\phi=0}$ contains both positive and negative values, one can construct a regular solution by gluing a numerical solution with a certain value of $p$ $=a^{\prime} /\left.a\right|_{\phi=0}$ to the $Z_{2}$ image of the solution with $a^{\prime} /\left.a\right|_{\phi=0}$ $=-p$. If the range does not contain both signs, one cannot construct a regular solution.

I performed the above procedure in five space-time dimensions $(n=5)$. From Eq. (9), the maximum value for the existence of an analytic solution is $v_{0}=3 / 5=9 / 15$. In fact, for $v_{0}=7 / 15$, I obtained $a^{\prime} /\left.a\right|_{\phi=0} \approx 0.0023,-0.015$ for $\phi_{c}$ $=0.2 \sqrt{3 / 2} \pi, 0.3 \sqrt{3 / 2} \pi$, respectively, and the existence of a regular solution is numerically supported. For $v_{0}=11 / 15$, however, the plotted values of $a^{\prime} /\left.a\right|_{\phi=0}$ in Fig. 4 indicate that $a^{\prime} /\left.a\right|_{\phi=0}$ takes only negative values. Thus it is numerically supported that there do not exist any regular de Sitter domain wall solutions in the parameter region (26).

\footnotetext{
${ }^{2}$ Namely, invariant under $\phi \rightarrow-\phi$.
} 


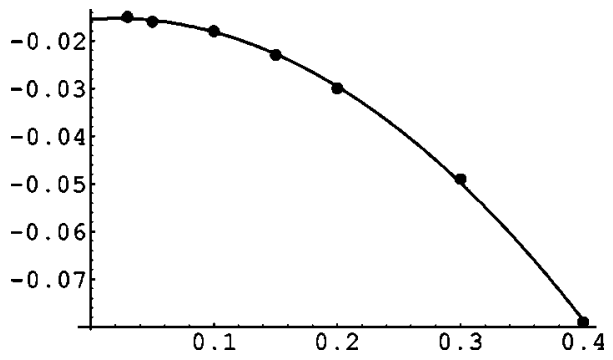

FIG. 4. The values of $a^{\prime} /\left.a\right|_{\phi=0}$ are plotted against $\phi_{c}$ for $v_{0}$ $=11 / 15, v_{1}=1, H=1$. The dots are the points where the numerical computations were performed, and the solid line is the fitting line with an assumed form $a^{\prime} /\left.a\right|_{\phi=0}=c_{0}+c_{1} \phi_{c}+c_{2} \phi_{c}^{2}$.

Qualitatively, in this parameter region, the constant part of the scalar field potential is so large that the expansion rate at the core is too large for a domain wall to keep its shape. Similar phenomena are discussed in the context of topological inflation in $[14,20,21]$. In [14], a perturbative analysis of the phase boundary between the existence and nonexistence of domain wall solutions for the four-dimensional system of gravity and a scalar field was performed, including the present case with an axion-like scalar field potential. ${ }^{3}$ According to the paper, the phase boundary is generally characterized by the equation

$$
\frac{3}{2}\left|V^{\prime \prime}(0)\right|-V(0)=0
$$

in our present notation for $n=4$. Substituting the parametrization (8), this becomes $2 v_{0}-v_{1}=0$, which agrees with Eq. (26) for $n=4$ (see Fig. 5).

Thus, in the region (26), the rapid expansion will ultimately sweep away the spatial dependence of the scalar field, and the dynamics will be mainly described by its timedependence. This will be the subject of Sec. VI.

\section{COSMOLOGICAL SOLUTIONS}

It is well known that, starting from a domain wall solution, a cosmological solution can be obtained by an analytic continuation which exchanges the transverse coordinate and the time coordinate [17]. An appropriate analytic continuation is given by

$$
\begin{gathered}
t \rightarrow i y, \\
y \rightarrow i t, \\
H \rightarrow i h,
\end{gathered}
$$

by which, a de Sitter domain wall solution (4) changes to a new solution with the substitution $y \rightarrow t$ and $H \rightarrow h$. This analytic continuation turns the de Sitter domain wall metric (2) into

\footnotetext{
${ }^{3}$ Denoted as a sine-Gordon potential in [14].
}

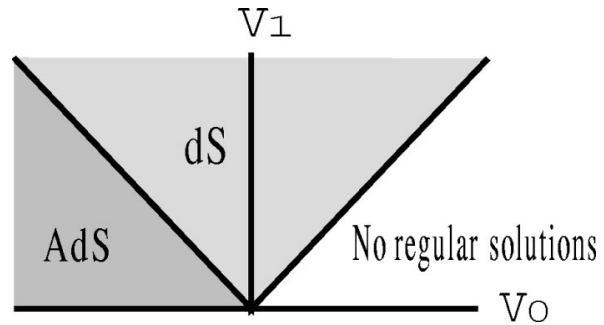

FIG. 5. The parameter regions for dS, AdS, and no regular domain wall solutions are shown. The two lines of boundaries are $n v_{0}+(n-2) v_{1}=n v_{0}-(n-2) v_{1}=0$. On the line $n v_{0}+(n-2) v_{1}$ $=0$, flat domain wall solutions exist.

$$
d s^{2}=-d t^{2}+a(t)^{2}\left(d y^{2}+e^{-2 h y} \sum_{i=1}^{n-2}\left(d x^{i}\right)^{2}\right)
$$

which describes a Friedmann-Robertson-Walker (FRW) cosmology of an open universe. Hence the new solution represents a finite lifetime open universe with a big-bang and a big-crunch.

Under the analytic continuation (28), the equations of motion (3) remain the same with the identification of the prime with the time derivative (overdot) and $H$ with $h$ and changing the sign of the scalar potential. Under the change of the sign of the potential, the region of the de Sitter solutions (9) is transformed to the identical region. Thus the parameter region for the open universe solution is the same as that of the de Sitter wall solutions (9) (see Fig. 6).

As for the anti-de Sitter solution, the analytic continuation (28) turns it into a cosmological solution of a closed universe. Because of the flip of the sign of the scalar potential, the parameter region of the closed universe solution is obtained by flipping Eq. (18) into

$$
(n-2) v_{1}<n v_{0}
$$

This agrees with the region where there are no regular domain wall solutions. The corresponding cosmological solutions are obtained by the substitution $y \rightarrow t$ and $H \rightarrow h$ in the AdS domain wall solutions (16). The periodic behavior of the AdS domain wall solutions is now interpreted as representing a cyclic universe.

It would be interesting to see the behavior of the energy density and the pressure. In a five-dimensional space-time, they are

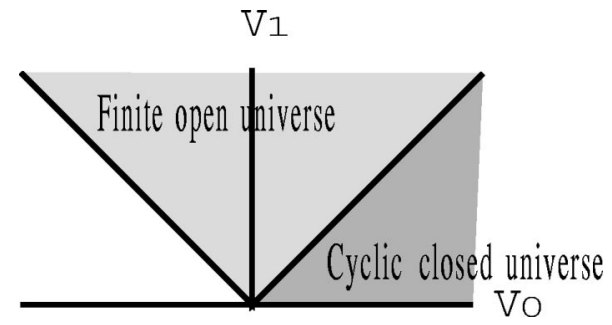

FIG. 6. The parameter regions for the two types of cosmological solutions are shown. The two lines of boundaries are $n v_{0}+(n$ -2) $v_{1}=n v_{0}-(n-2) v_{1}=0$. 

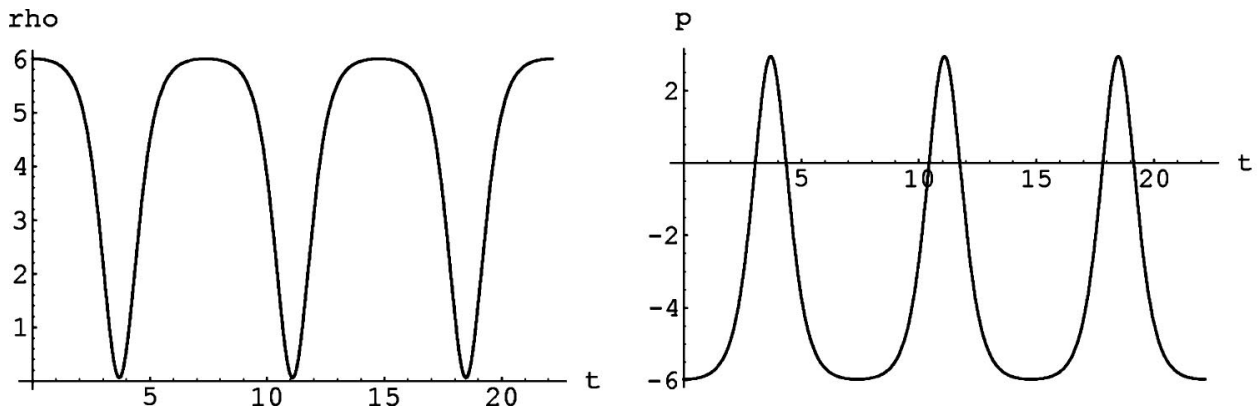

FIG. 7. The time evolution of the energy density and the pressure of the cosmological solution (five dimensions) corresponding to the AdS solution of Fig. 3. The energy density takes its maximum at the minimum of the scale factor to initiate the bounce, while the pressure takes its maximum at the maximum of the scale factor where the contraction begins.

$$
\begin{aligned}
\rho & =\frac{1}{4}(\dot{\phi})^{2}+\frac{1}{2} V(\phi) \\
& =6 h^{2}\left(1-\frac{\delta^{2}\left(1-\delta^{2}\right) \operatorname{sn}^{2}\left(h t, \sqrt{1-\delta^{2}}\right)}{\operatorname{dn}^{2}\left(h t, \sqrt{1-\delta^{2}}\right)}\right), \\
p & =\frac{1}{4}(\dot{\phi})^{2}-\frac{1}{2} V(\phi) \\
& =h^{2} \delta^{2}\left(-6-\frac{6}{\delta^{2}}+\frac{9}{\operatorname{dn}^{2}\left(h t, \sqrt{1-\delta^{2}}\right)}\right) .
\end{aligned}
$$

In Fig. 7 I plotted the time evolution of the energy density and the pressure for the cyclic universe solution with $h=1$ and $\delta=0.1$. At the minimum of the scale factor the energy density takes its maximum value and the pressure is approximately the minus of the energy density. At this period the large energy density initiates a bounce and the universe is at the inflation stage. On the other hand, at the maximum of the scale factor, the energy density is nearly vanishing and the pressure dominates. The large pressure works as a negative gravitational energy and initiates a contraction of the universe.

For a flat FRW universe, if I assume the null energy condition, the Hubble parameter satisfies an inequality

$$
\dot{H} \leqslant 0 \text {, }
$$

and there cannot exist a bounce. This also implies that, since our universe is expanding at present days, the universe must have started from a singularity. In the papers [22,23], some loopholes of the above discussion are presented from string theory to support the possibility of the pre-big-bang scenario [24]. In our cyclic solution, the existence of the positive curvature of the space changes the equation of motion of $H$ into

$$
\dot{H}=\frac{h^{2}}{a^{2}}-\frac{\dot{\phi}^{2}}{2(n-2)},
$$

and $\dot{H}$ can take both signs. Considering the high energy at the big-bang of the universe, it is plausible that some fluc- tuations of matters and gravity generate a region with a positive spatial curvature and the bounce of the universe simply happens from a classical dynamics. In this case, even though the present model seems far from what is the real universe, it might provide a simple toy model to study the pre-big bang scenario.

The inequality (32) also plays an important role in the $\mathrm{dS} /$ conformal field theory (CFT) correspondence. The central charge is given by an inverse power of the Hubble parameter, and the inequality (32) supports the interpretation of the time evolution as a renormalization group flow to UV [25,26]. In the case of a cyclic universe, however, the time flow cannot be interpreted in this way [27] because reverse process exists. If I take seriously the interpretation of $[25,26]$, there should be a kind of mechanism to prevent the above situation from happening. From this point, it would be interesting to try to consistently embed my simple model into string theory.

\section{SUMMARY AND DISCUSSIONS}

In this paper I have studied the analytic solutions of the system of gravity and a scalar field with an axionlike potential. They contain de Sitter thick domain walls, anti-de Sitter thick domain walls, finite lifetime universes with a big-bang and a big-crunch, and cyclic universes. These analytic solutions might be useful as toy models for the studies of the more general corresponding cases.

An obvious application of the analytic de Sitter domain wall solutions presented in this and previous papers [1] would be as toy models of our world through the brane world scenario. According to recent observations [28], our universe was in the inflation stage at the big-bang, and moreover, a tiny cosmological constant might exist even at present. In my model, gravity will be confined by the mechanism of [29], and it would be interesting to investigate the gravitational properties in such an accelerating domain wall universe.

Another interesting direction would be to embed my model into supersymmetric theories or superstring theory. The potential (8) has a simple form of an axion, which would be easily generated by field theory or string theory instanton corrections. According to [30], a de Sitter space-time cannot have any supersymmetries, and hence supersymmetries must be broken on a de Sitter domain wall. In the identification of 
our world with the domain wall, it seems challenging to explain the large hierarchy between the observed upper bound of the cosmological constant and the supersymmetry breaking scale. Based on a similar motivation, my model may be regarded as a gravity-coupled analogy of the SUSY-breaking domain wall solution presented in [31,32].

My cosmological solutions of cyclic universes might provide toy models for the scenario of $[33,34]$.

\section{ACKNOWLEDGMENTS}

The author would like to thank N. Sakai for discussions. The author was supported in part by Grant-in-Aid for Scientific Research (No. 12740150), and in part by Priority Area: "Supersymmetry and Unified Theory of Elementary Particles" (No. 707), from Ministry of Education, Science, Sports and Culture, Japan.
[1] N. Sasakura, J. High Energy Phys. 02, 026 (2002).

[2] M. Cvetic, S. Griffies, and S.J. Rey, Nucl. Phys. B381, 301 (1992).

[3] C. Csaki, J. Erlich, T.J. Hollowood, and Y. Shirman, Nucl. Phys. B581, 309 (2000).

[4] K. Skenderis and P.K. Townsend, Phys. Lett. B 468, 46 (1999).

[5] A. Chamblin and G.W. Gibbons, Phys. Rev. Lett. 84, 1090 (2000)

[6] O. DeWolfe, D.Z. Freedman, S.S. Gubser, and A. Karch, Phys. Rev. D 62, 046008 (2000).

[7] M. Gremm, Phys. Rev. D 62, 044017 (2000).

[8] E.E. Flanagan, S.H. Tye, and I. Wasserman, Phys. Lett. B 522, 155 (2001).

[9] E. Dudas and J. Mourad, Phys. Lett. B 486, 172 (2000).

[10] R. Blumenhagen and A. Font, Nucl. Phys. B599, 241 (2001).

[11] R. Gregory, Phys. Rev. D 54, 4955 (1996).

[12] P. Berglund, T. Hubsch, and D. Minic, Phys. Lett. B 534, 147 (2002).

[13] C. Charmousis, Class. Quantum Grav. 19, 83 (2002).

[14] F. Bonjour, C. Charmousis, and R. Gregory, Class. Quantum Grav. 16, 2427 (1999).

[15] F. Bonjour, C. Charmousis, and R. Gregory, Phys. Rev. D 62, 083504 (2000).

[16] A.H. Wang, Phys. Rev. D 66, 024024 (2002).

[17] See, for example, M. Cvetic and H.H. Soleng, Phys. Rep. 282, 159 (1997)

[18] O. DeWolfe and D.Z. Freedman, "Notes on fluctuations and correlation functions in holographic renormalization group flows," hep-th/0002226.
[19] S. Kobayashi, K. Koyama, and J. Soda, Phys. Rev. D 65, 064014 (2002).

[20] A. Vilenkin, Phys. Rev. Lett. 72, 3137 (1994).

[21] A.D. Linde, Phys. Lett. B 327, 208 (1994).

[22] J. Khoury, B.A. Ovrut, N. Seiberg, P.J. Steinhardt, and N. Turok, Phys. Rev. D 65, 086007 (2002).

[23] N. Seiberg, "From big crunch to big bang-is it possible?" hep-th/0201039.

[24] G. Veneziano, "String cosmology: The pre-big bang scenario," hep-th/0002094.

[25] A. Strominger, J. High Energy Phys. 11, 049 (2001).

[26] V. Balasubramanian, J. de Boer, and D. Minic, Phys. Rev. D 65, 123508 (2002).

[27] R. Argurio, "Comments on cosmological RG flows," hep-th/0202183.

[28] See, for example, M. Fukugita, "Cosmology and particle physics," hep-ph/0012214.

[29] L. Randall and R. Sundrum, Phys. Rev. Lett. 83, 4690 (1999).

[30] E. Witten, "Quantum gravity in de Sitter space," hep-th/0106109.

[31] N. Maru, N. Sakai, Y. Sakamura, and R. Sugisaka, "SUSY breaking by stable non-BPS walls," in C01-07-03.2, hep-th/0109087.

[32] N. Maru, N. Sakai, Y. Sakamura, and R. Sugisaka, "SUSY breaking by stable non-BPS configurations," hep-th/0112244.

[33] P.J. Steinhardt and N. Turok, "A cyclic model of the universe," hep-th/0111030.

[34] G.N. Felder, A. Frolov, L. Kofman, and A. Linde, Phys. Rev. D 66, 023507 (2002). 